Supporting Information for

\title{
Synthesis of Hindered Phenolic Esters over Ion-Exchange Resins
}

\author{
Wensheng Gao ${ }^{1}$, Ying Feng ${ }^{1}$, Yanyan Huang ${ }^{1}$, Ligong Chen ${ }^{1,2}$, Xilong Yan ${ }^{1,2^{*}}$ \\ ${ }^{1}$ School of Chemical Engineering and Technology, Tianjin University, Tianjin, P.R. China \\ ${ }^{2}$ Collaborative Innovation Center of Chemical Science and Engineering (Tianjin), Tianjin, P.R. \\ China \\ E-mail: yan@tju.edu.cn

\section{S1. General information of experiment}

The ion-exchange resins (NKC-9, D072, D001-CC, D001-SS, 001×4, 001×7, 001×14.5, D113), $\mathrm{HY}$ and $\gamma-\mathrm{Al}_{2} \mathrm{O}_{3}$ were obtained from the Chemical Plant of Nankai University (China) and the main parameters of the sulphonic acid functionalized resins were shown in Table S1. These catalysts were used after they have been dried under vacuum at $333 \mathrm{~K}$ for $48 \mathrm{~h}$. Other chemicals were purchased from commercial suppliers and used without further purification unless otherwise noted. HPLC were used to monitor the esterification. HPLC analyses were performed on a Agilent 1100 Series instrument at ambient temperature using Zorbaz Eclipse XDB-C18 column (reverse phase, $4.6 \mathrm{~mm} \times 250 \mathrm{~mm}$, 5-Micron), mobile phase: $90 \%$ methanol and aqueous acetic acid $(0.5 \%$, W/W). Column chromatography was performed using 200-300 mesh silica gel (Qingdao Haiyang). ${ }^{1} \mathrm{H}$ NMR and ${ }^{13} \mathrm{C}$ NMR spectra were recorded on Bruker AVANCE III 600MHz NMR spectrometer, using TMS as an internal standard. High-resolution mass spectra were measured with a Bruker Daltonics micrOTOF-Q II instrument (ESI). Elemental Analysis data were measured using an Elementar Vario Micro cube instrument. 
Table S1 Main parameters of sulphonic acid functionalized resins ${ }^{\mathrm{a}}$.

\begin{tabular}{cccccccc}
\hline Catalyst $^{\mathrm{b}}$ & Structure & $\begin{array}{c}\text { Acidity } \\
(\mathrm{mmol} / \mathrm{g})^{\mathrm{c}}\end{array}$ & $\begin{array}{c}\mathrm{S}_{\mathrm{BET}} \\
\left(\mathrm{m}^{2} / \mathrm{g}\right)\end{array}$ & $\begin{array}{c}\text { The ratio of } \\
\text { acidity and } \mathrm{S}_{\mathrm{BET}}\end{array}$ & $\begin{array}{c}\text { Pore volume } \\
\left(\mathrm{cm}^{3} / \mathrm{g}\right)\end{array}$ & $\begin{array}{c}\text { Average pore } \\
\text { diameter }(\mathrm{nm})\end{array}$ & $\mathrm{T}_{\max }\left({ }^{\circ} \mathrm{C}\right)$ \\
\hline NKC-9 & MR & 4.8 & 11.94 & 0.40 & 0.030 & 10.09 & 100 \\
D072 & MR & 4.4 & 15.79 & 0.28 & 0.044 & 11.03 & 120 \\
D001-CC & MR & 4.1 & 22.86 & 0.18 & 0.073 & 12.82 & 120 \\
D001-SS & MR & 4.2 & - & - & - & - & 120 \\
$001 \times 4$ & $\mathrm{G}$ & 4.5 & - & - & - & - & 120 \\
$001 \times 7$ & $\mathrm{G}$ & 4.5 & - & - & - & - & 120 \\
$001 \times 14.5$ & $\mathrm{G}$ & 3.8 & - & - & - & - & 120 \\
\hline
\end{tabular}

MR: macroporous structure. G: gel type polymer.

${ }^{\mathrm{a}}$ Date taken from reference ${ }^{[1]}$.

${ }^{\mathrm{b}}$ The particle size of ion-exchange resins is $0.3-1.25 \mathrm{~mm}$.

${ }^{\mathrm{c}}$ The acidity of dry ion-exchange resins was detected.

\section{S2. ${ }^{1} \mathrm{H}$ and ${ }^{13} \mathrm{C}$ NMR information of products:}

hexadecyl 3,5-di-tert-butyl-4-hydroxybenzoate ${ }^{[2]}$<smiles>CCOC(=O)c1cc(C(C)(C)C)c(O)c(C(C)(C)C)c1</smiles>

${ }^{1} \mathrm{H}$ NMR $\left(\mathrm{CDCl}_{3}, 600 \mathrm{MHz}\right): \delta=0.88\left(\mathrm{t}, \mathrm{J}_{\mathrm{HH}}=6.6 \mathrm{~Hz}, 3 \mathrm{H}, \mathrm{CH}_{3}\right), 1.25-1.43\left(\mathrm{~m}, 26 \mathrm{H}, \mathrm{C}_{13} \mathrm{H}_{26}\right)$, $1.46\left(\mathrm{~s}, 18 \mathrm{H}, 2 \mathrm{C}\left(\mathrm{CH}_{3}\right)_{3}\right), 1.75\left(\mathrm{~m}, 2 \mathrm{H}, \mathrm{CH}_{2}\right) 4.27\left(\mathrm{t}, \mathrm{J}_{\mathrm{HH}}=6.6 \mathrm{~Hz}, 2 \mathrm{H}, \mathrm{COOCH}_{2}\right), 5.64(\mathrm{~s}, 1 \mathrm{H}, \mathrm{OH})$, 7.9 (s, 2H; ArH).

${ }^{13} \mathrm{C} \mathrm{NMR}\left(\mathrm{CDCl}_{3}, 150 \mathrm{MHz}\right): \delta=14.13,22.71,26.11,28.85,29.32,29.39,29.60,29.72,30.16$, $31.95,34.33,64.68,121.60,127.00,135.67,158.05,167.21 \mathrm{ppm}$.

Elemental Analysis: Anal. Calcd for $\mathrm{C}_{31} \mathrm{H}_{54} \mathrm{O}_{3}$ : C, 78.43; H, 11.46; O, 10.11. Found: C, 78.44; H, $11.43 ; \mathrm{O}, 10.13$.

HRMS (ESI Positive) calc. for $\mathrm{C}_{31} \mathrm{H}_{54} \mathrm{O}_{3}$, [M+H] $]^{+}$475.4146, found 475.4146 . 

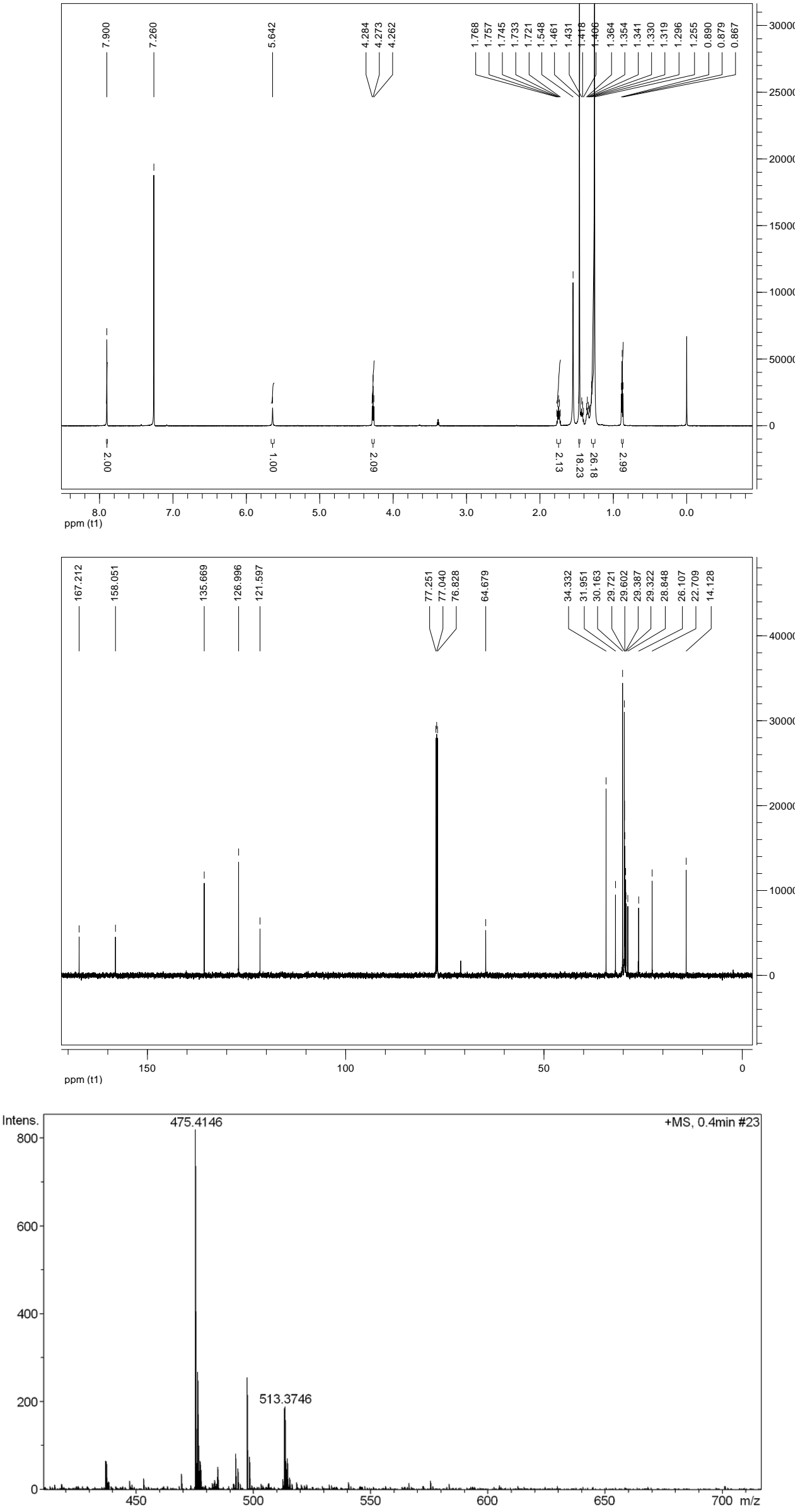
tetradecyl 3,5-di-tert-butyl-4-hydroxybenzoate<smiles>CCOC(=O)c1cc(C(C)(C)C)c(O)c(C(C)(C)C)c1</smiles>

${ }^{1} \mathrm{H}$ NMR $\left(\mathrm{CDCl}_{3}, 600 \mathrm{MHz}\right): \delta=0.88\left(\mathrm{t}, \mathrm{J}_{\mathrm{HH}}=6.6 \mathrm{~Hz}, 3 \mathrm{H}, \mathrm{CH}_{3}\right), 1.25-1.43\left(\mathrm{~m}, 22 \mathrm{H}, \mathrm{C}_{11} \mathrm{H}_{22}\right), 1.46(\mathrm{~s}$, $\left.18 \mathrm{H}, 2 \mathrm{C}\left(\mathrm{CH}_{3}\right)_{3}\right), 1.74\left(\mathrm{~m}, 2 \mathrm{H}, \mathrm{CH}_{2}\right), 4.28\left(\mathrm{t}, \mathrm{J}_{\mathrm{HH}}=6.6 \mathrm{~Hz}, 2 \mathrm{H}, \mathrm{COOCH}_{2}\right), 5.65(\mathrm{~s}, 1 \mathrm{H}, \mathrm{OH}), 7.91(\mathrm{~s}$, $2 \mathrm{H} ; \mathrm{ArH})$.

${ }^{13} \mathrm{C} \mathrm{NMR}\left(\mathrm{CDCl}_{3}, 150 \mathrm{MHz}\right): \delta=14.13,22.71,26.11,28.85,29.32,29.38,29.60,29.71,30.16$, $31.95,34.33,64.69,121.59,127.00,135.68,158.06,167.24 \mathrm{ppm}$.

Elemental Analysis: Anal. Calcd for $\mathrm{C}_{29} \mathrm{H}_{50} \mathrm{O}_{3}$ : C, 77.97; H, 11.28; O, 10.74. Found: C, 77.82; H, $11.29 ; \mathrm{O}, 10.89$

HRMS (ESI Positive) calc. for $\mathrm{C}_{29} \mathrm{H}_{50} \mathrm{O}_{3},[\mathrm{M}+\mathrm{H}]^{+}$447.3833, found 447.3833.

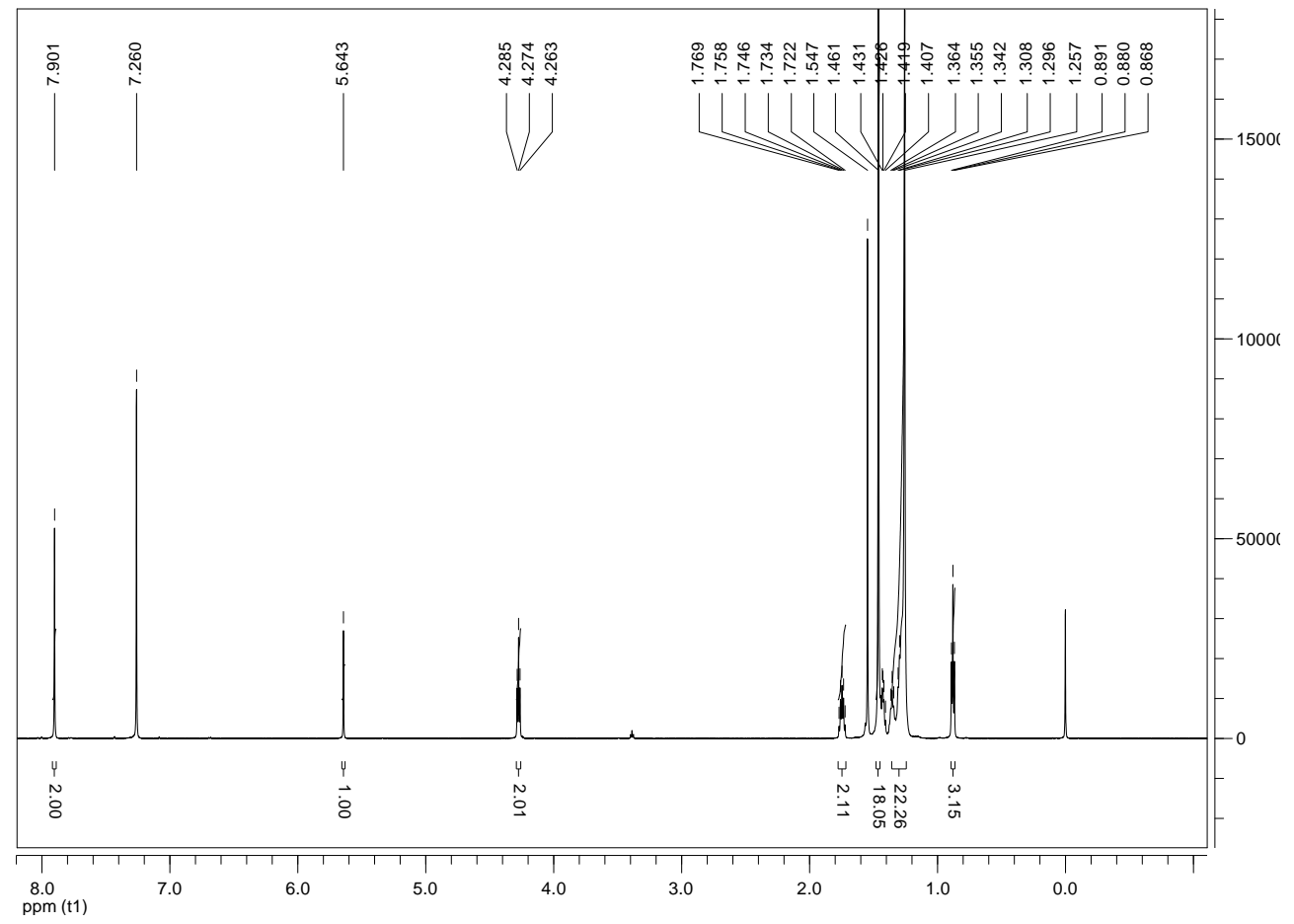



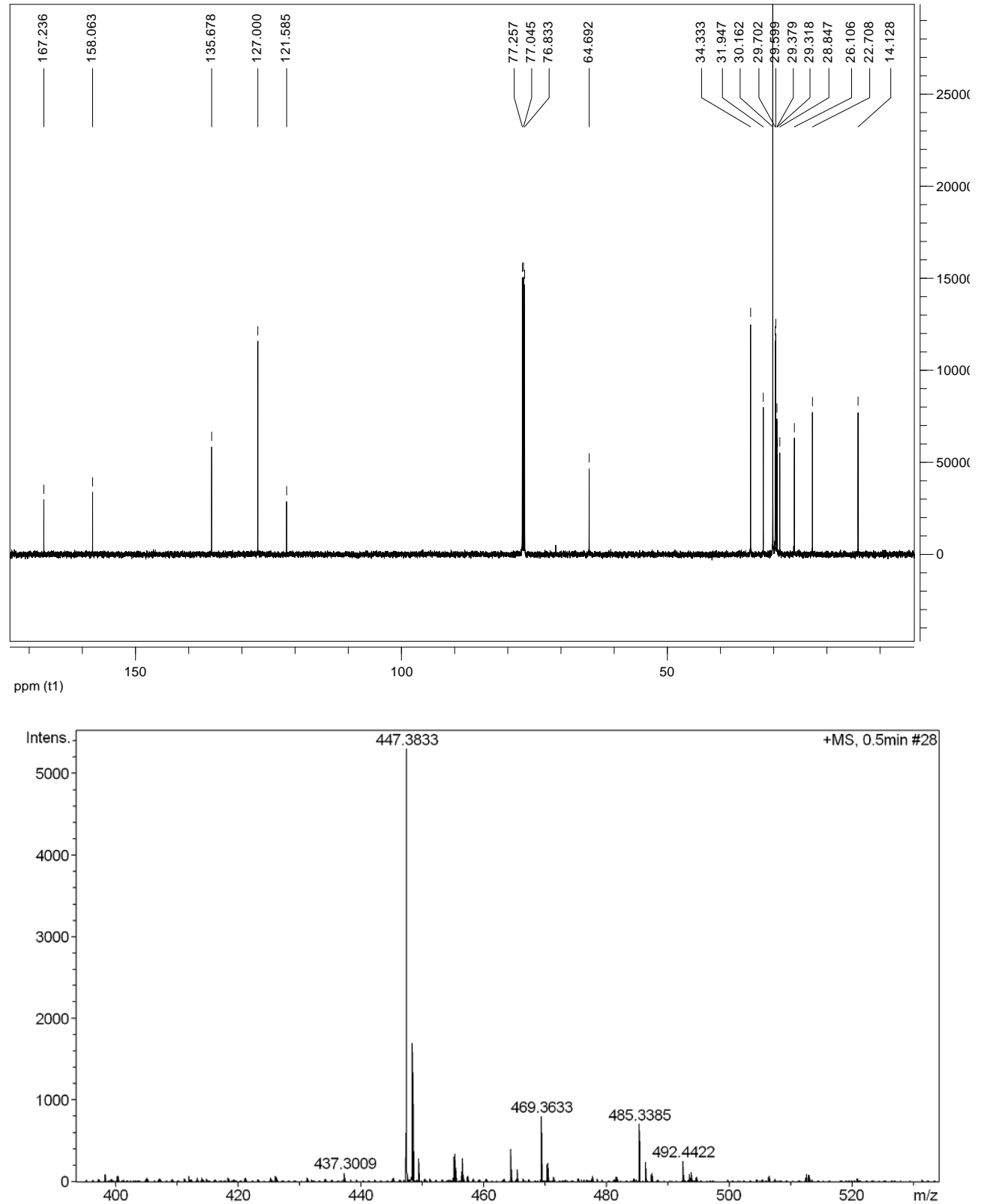

octyl 3,5-di-tert-butyl-4-hydroxybenzoate<smiles>CCCCCCOC(=O)c1cc(C(C)(C)C)c(O)c(C(C)(C)C)c1</smiles>

${ }^{1} \mathrm{H}$ NMR $\left(\mathrm{CDCl}_{3}, 600 \mathrm{MHz}\right): \delta=0.88\left(\mathrm{t}, \mathrm{J}_{\mathrm{HH}}=6.6 \mathrm{~Hz}, 3 \mathrm{H}, \mathrm{CH}_{3}\right), 1.28-1.44\left(\mathrm{~m}, 10 \mathrm{H}, \mathrm{C}_{5} \mathrm{H}_{10}\right), 1.46(\mathrm{~s}$, $\left.18 \mathrm{H}, 2 \mathrm{C}\left(\mathrm{CH}_{3}\right)_{3}\right), 1.74\left(\mathrm{~m}, 2 \mathrm{H}, \mathrm{CH}_{2}\right), 4.28\left(\mathrm{t}, \mathrm{J}_{\mathrm{HH}}=6.6 \mathrm{~Hz}, 2 \mathrm{H}, \mathrm{COOCH}_{2}\right), 5.65(\mathrm{~s}, 1 \mathrm{H}, \mathrm{OH}), 7.90(\mathrm{~s}$, $2 \mathrm{H} ; \mathrm{ArH})$. 
${ }^{13} \mathrm{C}$ NMR $\left(\mathrm{CDCl}_{3}, 150 \mathrm{MHz}\right): \delta=14.10,22.67,26.10,28.84,29.25,30.16,31.79,34.34,64.69$, $121.58,127.00,135.69,158.07,167.24 \mathrm{ppm}$.

Elemental Analysis: Anal. Calcd for $\mathrm{C}_{23} \mathrm{H}_{38} \mathrm{O}_{3}$ : C, 76.20; H, 10.56; O, 13.24. Found: C, 76.11; H, $10.60 ; \mathrm{O}, 13.28$.

HRMS (ESI Positive) calc. for $\mathrm{C}_{23} \mathrm{H}_{38} \mathrm{O}_{3},[\mathrm{M}+\mathrm{H}]^{+}$363.2894, found 363.2894.
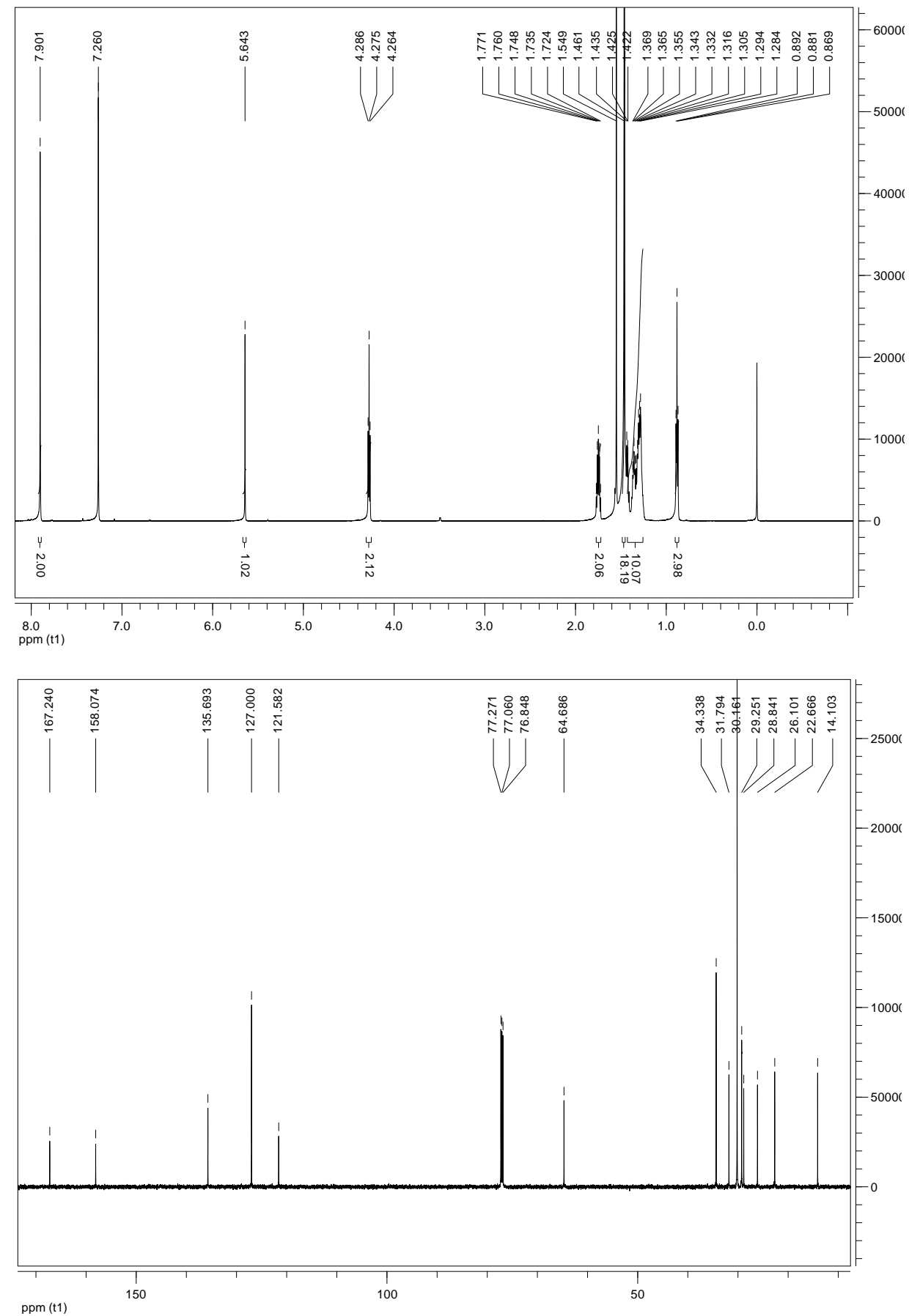


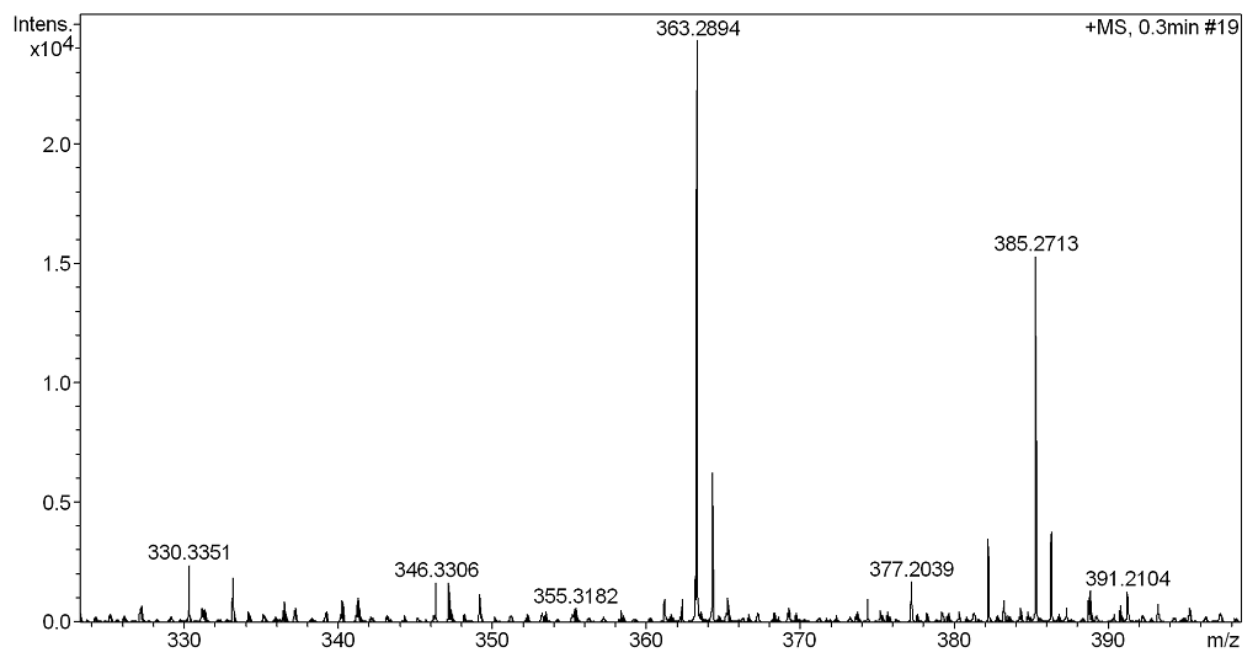

hexadecyl 3-(tert-butyl)-4-hydroxybenzoate ${ }^{[3]}$<smiles>CCOC(=O)c1ccc(O)c(C(C)(C)C)c1</smiles>

${ }^{1} \mathrm{H} \mathrm{NMR}\left(\mathrm{CDCl}_{3}, 600 \mathrm{MHz}\right): \delta=0.88\left(\mathrm{t}, \mathrm{J}_{\mathrm{HH}}=7.2 \mathrm{~Hz}, 3 \mathrm{H}, \mathrm{CH}_{3}\right), 1.25-1.41\left(\mathrm{~m}, 26 \mathrm{H}, \mathrm{C}_{13} \mathrm{H}_{26}\right), 1.42(\mathrm{~s}$, $\left.9 \mathrm{H}, \mathrm{C}\left(\mathrm{CH}_{3}\right)_{3}\right), 1.75\left(\mathrm{~m}, 2 \mathrm{H}, \mathrm{CH}_{2}\right), 4.28\left(\mathrm{t}, \mathrm{J}_{\mathrm{HH}}=6.6 \mathrm{~Hz}, 2 \mathrm{H}, \mathrm{COOCH}_{2}\right), 5.70(\mathrm{~s}, 1 \mathrm{H}, \mathrm{OH}), 6.71(\mathrm{~d}$, $\left.\mathrm{J}_{\mathrm{HH}}=7.8,1 \mathrm{H}, \operatorname{ArH}\right), 7.78\left(\mathrm{dd}, \mathrm{J}_{\mathrm{HH}}=1.8\right.$ and $\left.8.4 \mathrm{~Hz}, 1 \mathrm{H}, \operatorname{ArH}\right), 8.00\left(\mathrm{~d}, \mathrm{~J}_{\mathrm{HH}}=1.8 \mathrm{~Hz}, 1 \mathrm{H}, \operatorname{ArH}\right)$.

${ }^{13} \mathrm{C} \mathrm{NMR}\left(\mathrm{CDCl}_{3}, 150 \mathrm{MHz}\right): \delta=14.13,22.71,26.08,28.79,29.32,29.36,29.61,29.68,29.72$, $31.95,34.67,65.11,116.36,121.99,129.25,136.17,159.29,167.7 \mathrm{ppm}$.

Elemental Analysis: Anal. Calcd for $\mathrm{C}_{27} \mathrm{H}_{46} \mathrm{O}_{3}: \mathrm{C}, 77.46 ; \mathrm{H}, 11.07$; O, 11.46. Found: C, 77.41; H, 11.09; O, 11.49 .

HRMS (ESI Positive) calc. for $\mathrm{C}_{27} \mathrm{H}_{46} \mathrm{O}_{3}$, [M+H] $]^{+}$419.3520, found 419.3521 . 

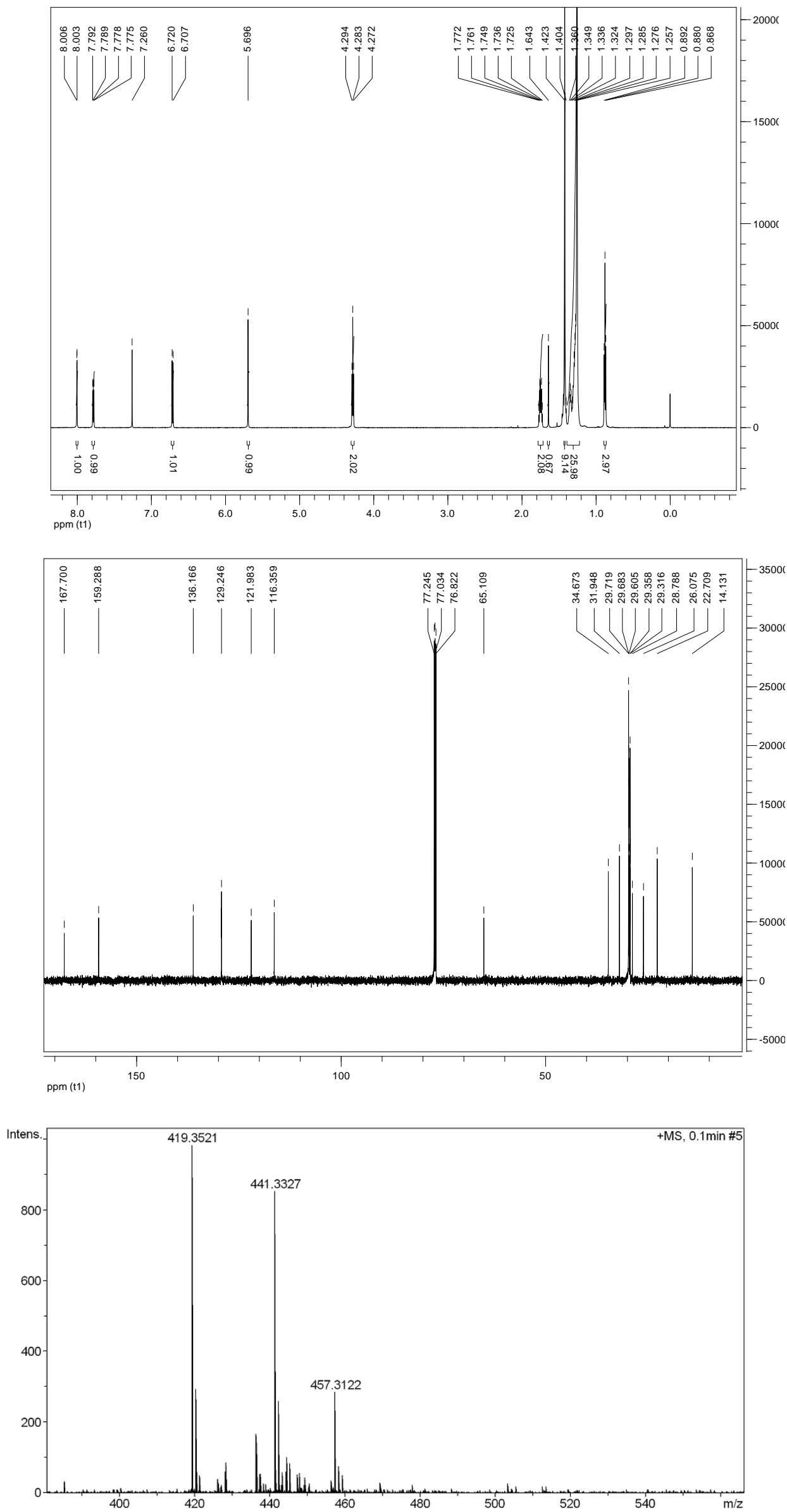
hexadecyl 4-hydroxybenzoate ${ }^{[3]}$<smiles>CCOC(=O)c1ccc(O)cc1</smiles>

${ }^{1} \mathrm{H} \mathrm{NMR}\left(\mathrm{CDCl}_{3}, 600 \mathrm{MHz}\right): \delta=0.86\left(\mathrm{t}, \mathrm{J}_{\mathrm{HH}}=7.2 \mathrm{~Hz}, 3 \mathrm{H}, \mathrm{CH}_{3}\right), 1.25-1.46\left(\mathrm{~m}, 26 \mathrm{H}, \mathrm{C}_{13} \mathrm{H}_{26}\right), 1.74(\mathrm{~m}$, $\left.2 \mathrm{H}, \mathrm{CH}_{2}\right), 4.28\left(\mathrm{t}, \mathrm{J}_{\mathrm{HH}}=6.6 \mathrm{~Hz}, 2 \mathrm{H}, \mathrm{COOCH}_{2}\right), 5.47(\mathrm{~s}, 1 \mathrm{H}, \mathrm{OH}), 6.86\left(\mathrm{dd}, \mathrm{J}_{\mathrm{HH}}=2.4\right.$ and $7.2 \mathrm{~Hz}$, $2 \mathrm{H} ; \mathrm{ArH}), 7.96\left(\mathrm{dd}, \mathrm{J}_{\mathrm{HH}}=3\right.$ and $\left.9.6 \mathrm{~Hz}, 2 \mathrm{H} ; \mathrm{ArH}\right)$.

${ }^{13} \mathrm{C} \mathrm{NMR}\left(\mathrm{CDCl}_{3}, 150 \mathrm{MHz}\right): \delta=14.13,22.70,26.06,28.74,29.31,29.38,29.55,29.61,29.67$, $29.71,31.94,65.26,115.31,122.47,131.93,160.43,167.26 \mathrm{ppm}$.

Elemental Analysis: Anal. Calcd for $\mathrm{C}_{23} \mathrm{H}_{38} \mathrm{O}_{3}$ : C, 76.20; H, 10.56; O, 13.24. Found: C, 76.22; H, $10.60 ; \mathrm{O}, 13.18$.

HRMS (ESI Positive) calc. for $\mathrm{C}_{23} \mathrm{H}_{38} \mathrm{O}_{3}$, [M+Na] $]^{+}$385.2713, found 385.2711.

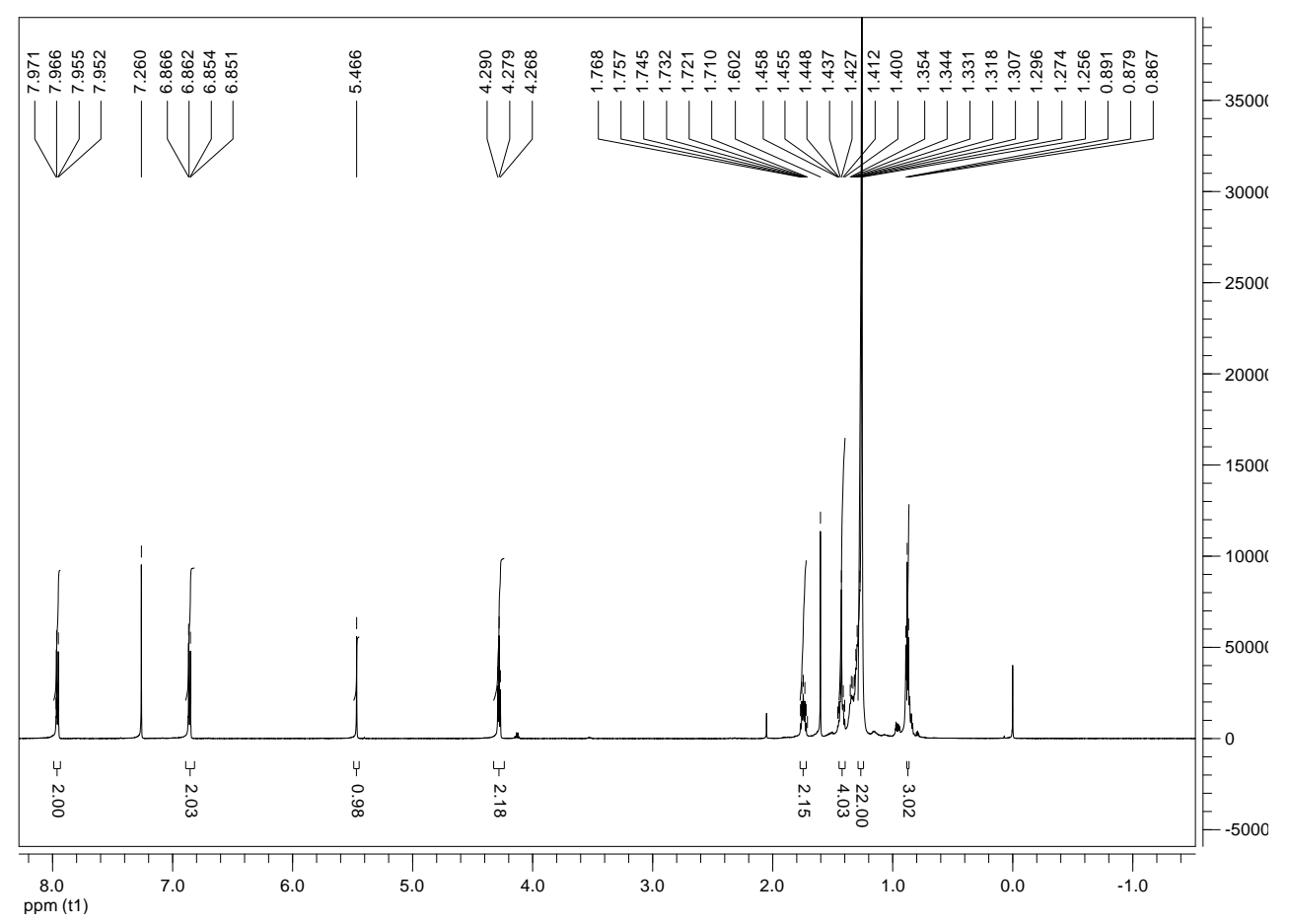



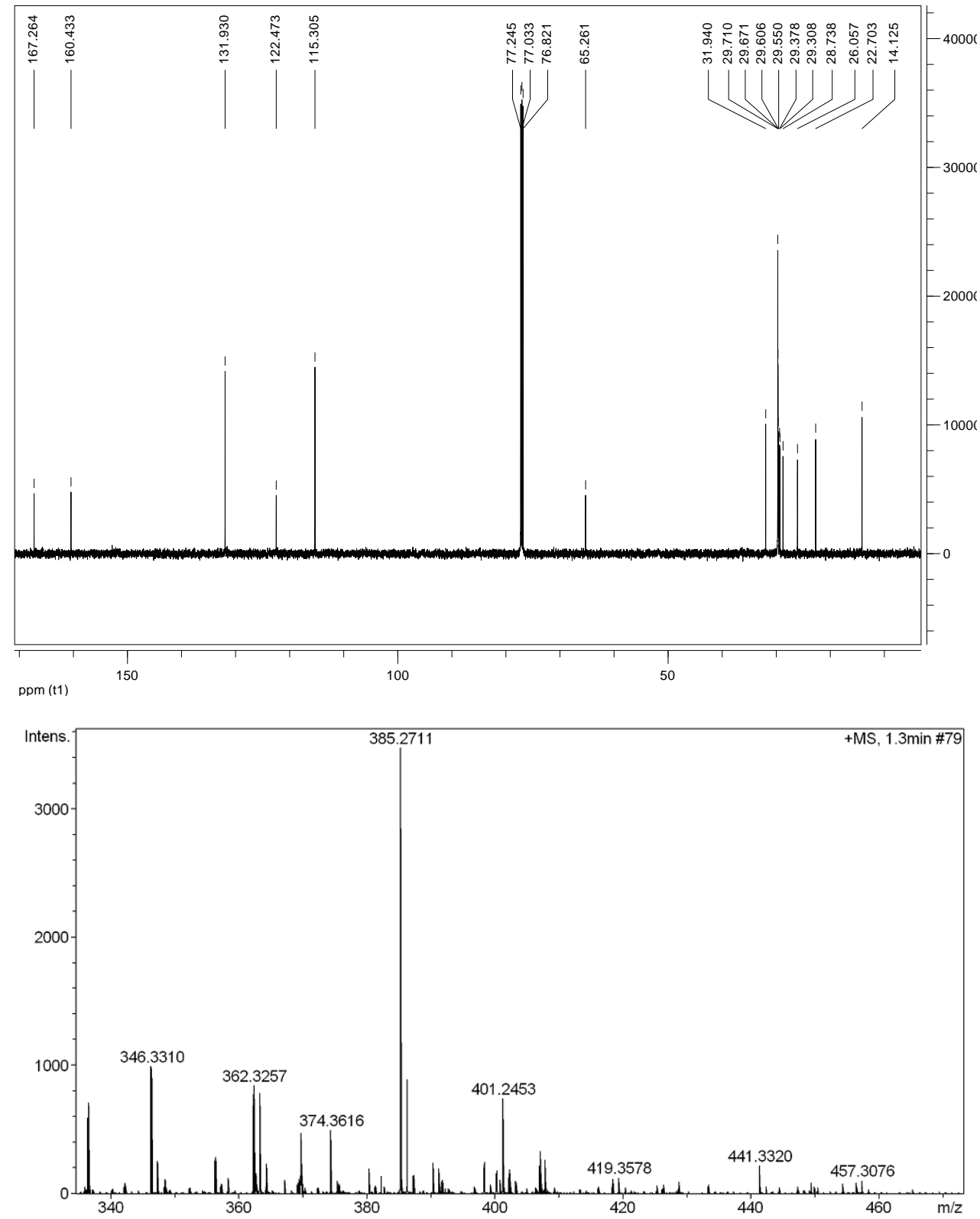

\section{Reference}

[1] Wang, W.; Wei, R.; Yin, G.; Tian, J.; Duan, Y.; Chen, L.; Li, Y. The synthesis of asymmetric ethylenediamine derivatives catalyzed by ion-exchange resins. Res. Chem. Intermediat. 2014, 1-12.

[2] Jordan, S.; Taylor, L. HPLC separation with solvent elimination FTIR detection of polymer additives. J. chromatogr. sci. 1997, 35, 7-13.

[3] Ohkatsu, Y.; Takenaka, H.; Kamiyama, N. Interaction of Benzoate-type Ultraviolet Absorbers with Hindered Amine Light Stabilizers. J. Jpn. Petrol. Inst. 2008, 51, 95-101. 